\title{
Estandarización del modelo langendorff de corazón aislado para evaluar los daños inducidos por isquemia-reperfusión
}

\author{
Mero, Aldahir \\ Departamento de Farmacología, Facultad de Medicina, Universidad de Panamá \\ Ciudad de Panamá, Panamá \\ aldahirmero_20@hotmail.com \\ Guerrero, Estela \\ Departamento de Farmacología, Facultad de Medicina, Universidad de Panamá \\ Ciudad de Panamá, Panamá \\ ORCID: 0000-0002-0029-1827
}

\section{Diaz, Maricselis}

Centro de investigaciones Psicofarmacológicas, Facultad de Medicina, Universidad de Panamá. Ciudad de

Panamá, Panamá

maricselis@hotmail.com

\section{Morales, Abdy}

Departamento de Farmacología, Facultad de Medicina, Universidad de Panamá

Ciudad de Panamá, Panamá

moba245@gmail.com

Morán-Pinzón, Juan

Departamento de Farmacología, Facultad de Medicina, Universidad de Panamá

Ciudad de Panamá, Panamá

coljamp@gmail.com

Hernandez, Xenia

Departamento de Farmacología, Facultad de Medicina, Universidad de Panamá

Ciudad de Panamá, Panamá

xeniagiselle12@gmail.com

\section{Abstract}

The ischemia-reperfusion model is an experimental pathway to reproduce the characteristics of an acute cardiac ischemic event. Our aim was to validate the Langendorff isolated rat heart model. The hearts were harvested and connected to a perfusion system to assess 
tissue viability, and we performed two perfusion rate protocols: 10.5 and $12.0 \mathrm{~mL} / \mathrm{min}$. On the other hand, the ischemia induction was assessment by perfusion suppressing or oxygen deprivation in the perfused solution. Cardiac parameters were obtained at 30 minutes, just before ischemia was induced (suspension of perfusion or oxygen deprivation). These parameters were also obtained at 70 and 120 minutes of the experiment. In all cases, a control group was used, where perfusion and oxygenation were kept continuous. For the determination infarct area (percentage), we found that this value was approximately $5 \%$ for both controls. In suspension-induced ischemia group by of perfusion at $10.5 \mathrm{~mL} / \mathrm{min}$ rate was $22 \%$ meanwhile $38 \%$ was observed at $12 \mathrm{~mL} / \mathrm{min}$ rate. When the ischemia was induced by oxygen deprivation, we observed that at 10.5 and $12 \mathrm{~mL} / \mathrm{min}$ perfusion rate, the infarct areas was $44 \%$ and $58 \%$, respectively. We conclude that tissue viability is more stable at a perfusion rate of $10.5 \mathrm{~mL} / \mathrm{min}$, and that ischemia is more reliably when oxygenation is supressed.

Keywords: Standardization, ischemia, reperfusion, Langendorff, infarct area.

\section{Resumen}

El modelo de isquemia-reperfusión representa una vía experimental para reproducir las características de un evento isquémico cardiaco agudo. Nuestro objetivo era validar el modelo Langendorff en corazón aislado de rata. Extrajimos el corazón y lo conectamos a un sistema de perfusión para evaluar la viabilidad del tejido, en donde modificamos la velocidad de perfusión (10.5 y $12.0 \mathrm{~mL} / \mathrm{min}$ ) y por otra parte, la inducción de la isquemia, que se realizó mediante la suspensión de la perfusión o la deprivación del oxigeno en la solución perfundida. Los parámetros cardiacos controles se obtuvieron justo antes de generar la isquemia y los 70 y 120 minutos de iniciada la perfusión. En todos los casos, mantuvimos un grupo control donde la perfusión fue continua, igual que la oxigenación del tejido cardiaco. En la determinacion del \% de área de infarto se encontró que este valor fue de aproximadamente $5 \%$ para ambos controles. En la isquemia inducida por la suspension de la perfusion la velocidad de 10.5 fue de $22 \%$ y un $38 \%$ en la velocidad de 12 $\mathrm{mL} / \mathrm{min}$. Cuando la isquemia fue inducida por deprivación de oxigeno los grupos de $10.5 \mathrm{y}$ $12 \mathrm{ml} / \mathrm{min}$ indujeron un $44 \%$ y $58 \%$, respectivamente.Logramos concluir que la viabilidad del tejido es más estable cuando se perfunde a una velocidad de $10.5 \mathrm{~mL} / \mathrm{min}$, y que la isquemia se alcanza con mayor fiabilidad cuando se interrupe de la oxigenación del tejido.

Palabras claves: Estandarización, isquemia, reperfusion, Langendorff, área de infarto 


\section{INTRODUCCIÓN}

Podemos denominar al método de Langendorff de corazon aislado, como la perfusión de las arterias coronarias de un corazón por una solución nutricia y oxigenada que fluye retrogradamente desde la aorta [1]. Este método fue establecido en 1897 por Oscar Langendorff y sus experimentos se llevaron a cabo principalmente utilizando corazones de gatos, pero también de perros y conejos. [2]. Con el tiempo el método de Langendorff de corazón aislado se ha convertido en el modelo estandar para la enseñanza y la investigación cardiovascular básica, realizando aportes sobre temas como la regulación de la función cardíaca, flujo coronario y el metabolismo cardíaco [3].

En este modelo, el corazón extraído de un animal anestesiado, se canula y conecta a un aparato de perfusión. El sistema permanece conectado a un bomba que perfunde la solucion nutricia, burbujeada continuamente con carbógeno $\left(95 \% \mathrm{O}_{2}\right.$ y $\left.5 \% \mathrm{CO}_{2}\right)$, desde un reservorio, pasando a traves de tubos termostatizados a $37^{\circ} \mathrm{C}$, hasta el tejido aislado [1].

A partir este modelo obtenemos parámetros fisiológicos, morfológicos y bioquímicos, que pueden ser modificados a través de intervenciones farmacológicas. Adicionalmente, el modelo de Langendorff permite fácilmente derivaciones protocolares para reproducir procesos isquémicos y arritmias, lo que tambien permite valorar las acciones de farmacos y diferentes compuestos sobre estas patologías. (4).

Entre las desventajas de este método cuentan que la preparación requiere habilidad y delicadeza debido que el corazón es vulnerable a las contusiones. [2].

\section{MÉTODO}

\section{A. Modelo animal}

El estudio utilizó ratas Wistar albinas con un peso entre de 250 a 300 g, provenientes del Bioterio del Instituto de Investigaciones Científicas de Alta Tecnología (INDICASAT-AIP). Los animales se mantuvieron a una temperatura de $21 \pm 2^{\circ} \mathrm{C}$ y con un ciclo normal de 12 horas de luz/oscuridad. Este estudio fue realizado siguiendo las normas de manejo de animales recogida en la guia titulada "Guide for the care and use of laboratory animals" $y$ el protocolo fue aprobado por el Comité de Bioetica de la Universidad de Panama ( Nota No CBUP/153/2020).

\section{B. Extirpación del corazón}

Los animales fueron anestesiados con pentobarbital sódico $(50 \mathrm{mg} / \mathrm{kg}$ ) y anticoagulados con heparina sódica (500 U/kg). Una vez comprobado el plano anestésico en el animal, abrimos la cavidad abdominal realizando una incisión transversal. El diafragma se seccionó y se hicieron incisiones laterales a lo largo de la caja torácica. El corazón se separó, haciendo 
un solo corte con las tijeras a través de este punto y del tejido conectivo que fija al corazón. Seguidamente, el corazón se colocó en un vaso que contenía solución amortiguadora de Krebs-Henseleit helada. Por ultimo, la aorta del corazón se canuló y se conectó al sistema de perfusión. [5].

\section{Modificaciones protocolares para controlar viabilidad e inducir la isquemia}

Entra las variables modificadas están la velocidad de perfusión, que se ha relacionado con la viabilidad del corazón. Con base a estudios previos (datos no publicados), seleccionamos las velocidades de 10.5 y $12 \mathrm{ml} / \mathrm{min}$ para ser empleadas en la perfusión del corazón aislado. Considerando ambas velocidades, establecimos grupos controles y grupos en los que se induce isquemia reperfusión. La inducción de la isquemia, que se relaciona con el daño asociado a la isquemia reperfusión, se produjo mediante la suspensión de la perfusión (SP) o por la deprivación de oxígeno (DO) en la solución perfundida.

En todos los protocolos se mantiene un grupo control, donde los corazones son perfundidos de forma continua y con solucion oxigenada durante 120 minutos, mientras que, el grupo isquemia reperfusión, se estabiliza perfundiendolo durante los primeros 30 min, seguido por un periodo de isquemia por SP o DO de 30 min., para proceder al periodo de reperfusión durante una hora.

\section{Determinación de los parámetros cardiacos}

El registro de los datos fue por medio de un balón de látex, insertado en el ventrículo izquierdo a través de la válvula mitral y conectándolo a un transductor de presión. Para todos los corazones, cambios en la presión desarrollada en el ventrículo izquierdo (PDVI) y la frecuencia cardíaca (FC) se registraron continuamente utilizando un sistema de adquisición de datos PowerLab v8. Además, se logró calcular otros valores de la funcionalidad cardiaca como lo son el MaxdP/dt (indicador de la contracción miocárdica), MindP/dt (indicador de la relajación miocárdica) y el doble producto que nos indica el trabajo cardiaco total. [6].

\section{E. Determinaciones histológicas del corazón}

Una vez concluida la fase de $I / R$, los corazones fueron sometidos a un proceso de fijación y tinción. El corazón se congeló y obtuvimos secciones desde el ápice hacia la base del corazón, las cuales fueron tratadas con TTC al 1\% durante 15-20 minutos y se fijaron con formaldehído al 10\% durante $20 \mathrm{~min}$. Las secciones teñidas se fotografiaron bajo estereosopio y, utilizando el software Image ${ }^{\circledR}$, se trazaron las zonas de infarto y se calcularon las áreas respectivas en términos de píxeles [ 7]. 


\section{RESULTADOS}

\section{A. Determinación de los parámetros cardiacos}

Cuando el tejido fue perfundido a la velocidad de $10,5 \mathrm{~mL} / \mathrm{min}$, observamos que la presión desarrollada por el ventrículo izquierdo (PDVI), se mantuvo constante a lo largo del experimento con valores aproximados de $80,00 \mathrm{mmHg}$. Sin embargo, la perfusión a una velocidad de $12 \mathrm{~mL} / \mathrm{min}$ genera un estrés que empobrece el desarrollo de la PDVI y observamos que esta se reduce en el tiempo y pasa de un valor control de 79,01 a 61,14 $\mathrm{mmHg}$ a los 120 minutos. En contraste, la viabilidad del tejido se mantiene cuando se perfunde a $10,5 \mathrm{~mL} / \mathrm{min}(79,06$ a $82,36 \mathrm{mmHg}$ control y 120 minutos, respectivamente). Autores como Liu y col. (2018) reportan valores de PDVI similares a los obtenidos en nuestro estudio [8]. Al valorar el impacto que sobre este parámetro poseen las intervenciones para generar I/R, observamos que la DO redujo la PDVI. En este grupo el valor control, antes de inducir la isquemia, fue de 95,71 $\pm 11,87 \mathrm{mmHg}$ y disminuyó hasta 47,52 $\pm 10,65 \mathrm{mmHg}$ al minuto 70 cuando la perfusión se realizo a 10,5 mL/min. (Fig 1A).

El doble producto (DP) se encuentra disminuido en los procesos de isquemia reperfusión [9] y esto quedó demostrado en la DO del corazón perfundido a la velocidad de $10.5 \mathrm{~mL} / \mathrm{min}$, en donde, al minuto 70, disminuyó hasta un valor de $10361 \pm 2260$, en comparación con el control que mantiene un valor de $22391 \pm 2286$ (Fig 1C). En la figura 1D se representan los resultados del doble productos para corazones sometidos a una perfusión a $12 \mathrm{~mL} /$ min. Evaluando este parámetro, volvemos a observar como el estrés inducido por una elevada velocidad de perfusión genera una reducción del trabajo cardiaco en el tiempo, enmascarando el efecto de la inducción de la isquemia por DO (Fig 2). Autores como Liu y col (2018) reportan valores de DP parecidos a los nuestros [8].

Durante la isquemia se genera una disfunción y muerte de los cardiomiocitos empobreciendo la contractilidad, parámetro que en nuestro estudio es valorado a través de la determinación del Max dP/dt. Con la perfusion de 10,5 y $12 \mathrm{~mL} /$ min, al minuto 70 y en los protocolos de $\mathrm{DO}$, se refleja una reduccion significativa del Max dP/dt, hasta valores de 1580,71 $\pm 365,48$ y 1488,26 $\pm 318,38 \mathrm{mmHg} / \mathrm{s}$, respectivamente, frente a los valores promedios desarrollados en el control (2978 $\pm 262 \mathrm{mmHg} / \mathrm{s}$ ). Este hallazgo a todas luces es un dato indicativo del proceso isquémico (Fig 2A y 2B). (Fig 3). Hallazgos similares han sido publicados por otros autores [10].

La relajación miocardica es un parámetro relacionado al Min dP/dt, que al igual que el Max dP/dt se afectan en los procesos isquemico cardiacos. En nuestro estudio, quedo registrado una reducción de este parámetro al minuto 70 en el protocolo de $\mathrm{DO}$, alcanzando 
un valor de $-969,97 \pm 180,59 \mathrm{~mm} \mathrm{Hg} / \mathrm{s}$. mientras que el valor del control fue de $-1831 \pm 190$ $\mathrm{mm} \mathrm{Hg} / \mathrm{s}$.(Fig 2C y 2D) Se han publicado valores de Min dP/dt parecidos a los obtenidos por nosotros [11].
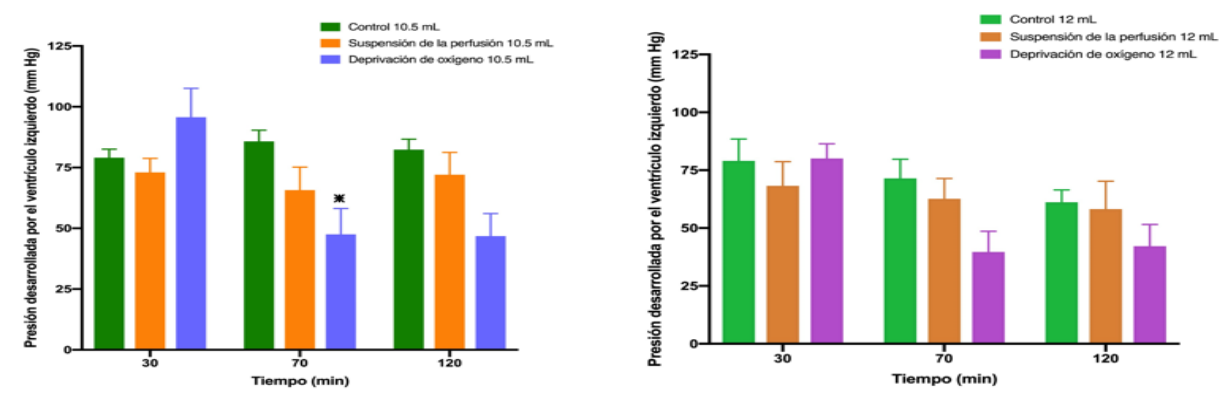

1.

RESULTADOS
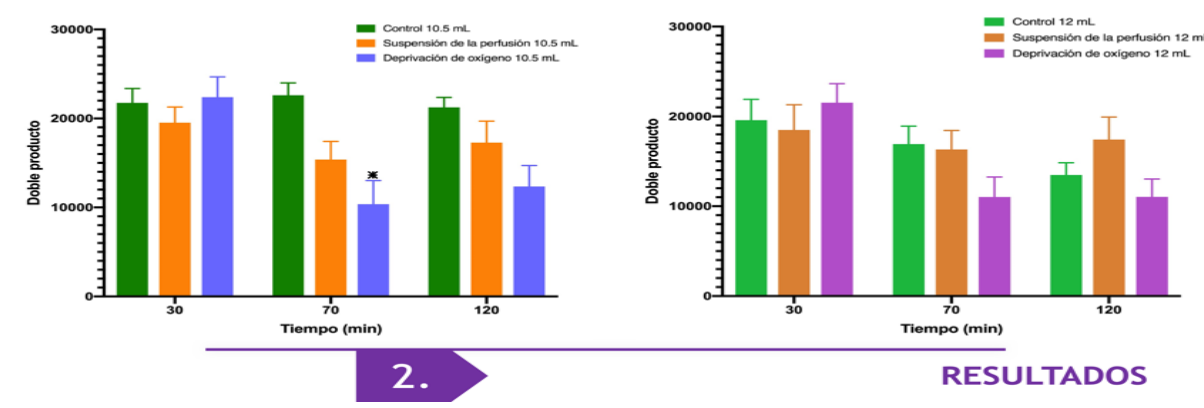

Figura 1. Efecto del tipo de inducción de la isquemia sobre la presión desarrollada por el ventriculo izquierdo en corazones perfundidos a $10,5 \mathrm{~mL} / \mathrm{min}$ (A) y 12,0 mL/min (B); y sobre el doble producto cuando la velocidad de perfusion fue de $10,5 \mathrm{~mL} / \mathrm{min}$ (C) y $12,0 \mathrm{~mL} / \mathrm{min}$ (D) * $p<0,05$ frente al control.
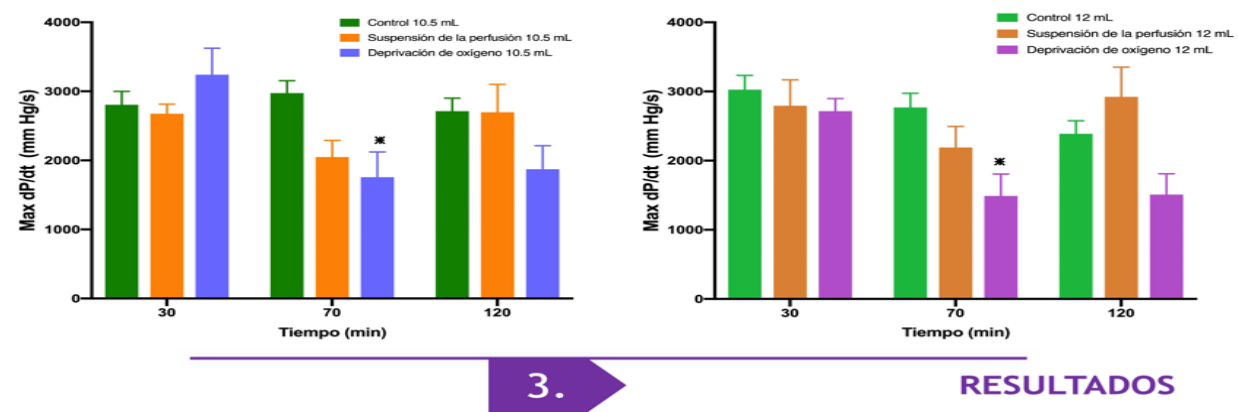

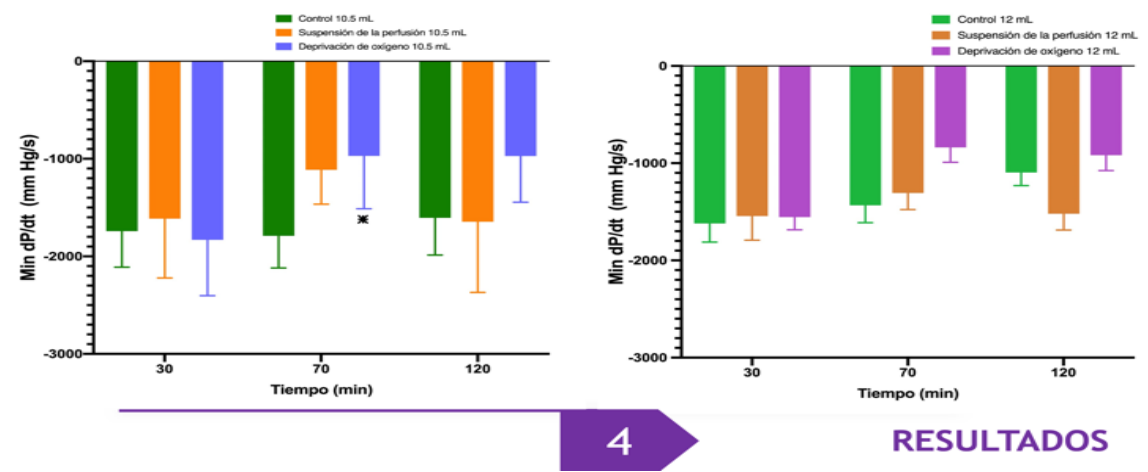

Figura 2. Efecto del tipo de inducción de la isquemia sobre el Max $\mathrm{dP} / \mathrm{dt}$ en corazones perfundidos a $10,5 \mathrm{~m} \mathrm{~L} / \mathrm{min}$ (A) y $12,0 \mathrm{ml} / \mathrm{min}$ (B); y sobre el Min dP/dt cuando la velocidad de perfusion fue de 10,5 $\mathrm{mL} / \mathrm{min}$ (C) y $12,0 \mathrm{~mL} / \mathrm{min}$ (D) * $\mathrm{p}<0,05$ frente al control.

\section{B. Determinaciones histológicas del corazón}

Los corazones controles evidenciaron un $6 \%$ de área de infarto. Cuando se inducía la isquemia por SP en la velocidad de $10,5 \mathrm{~mL} / \mathrm{min}$ se generó un $22 \%$ y un $38 \%$ de área de infarto en la velocidad de $12 \mathrm{~mL} / \mathrm{min}$. En cambio el protocolo de DO los grupos de 10,5 y 12 indujeron un $44 \%$ y $58 \%$ de area de infarto, respectivamente. .(Fig 3)

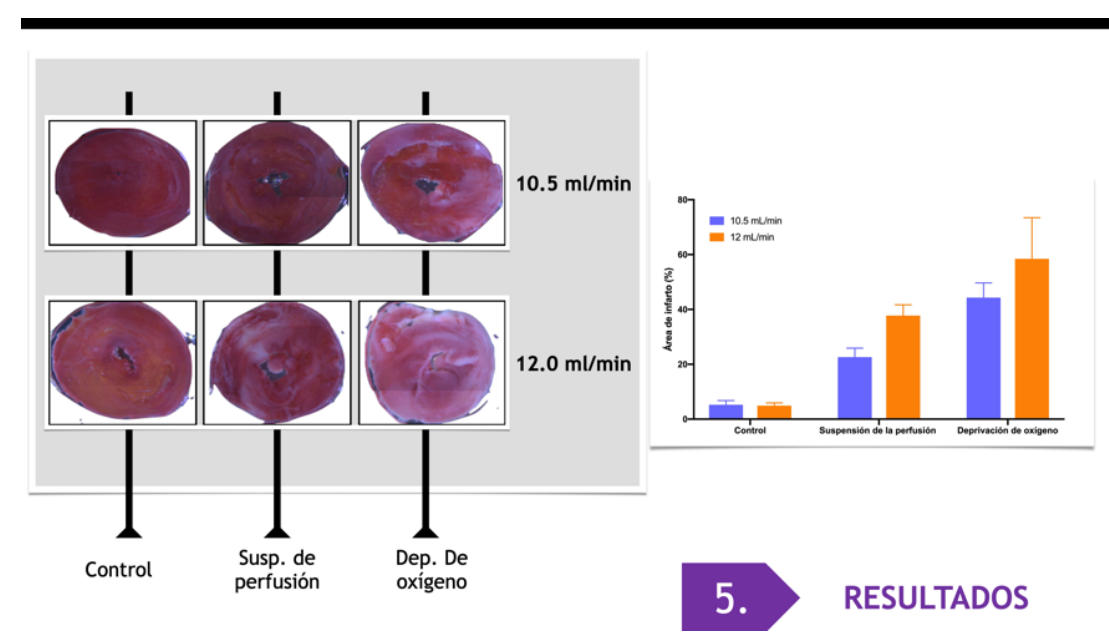

Figura 3. Cortes histológicos teñidos con TTC para determinar el área de infarto 


\section{CONCLUSIONES}

Se ha estandarizado una vía experimental válida para reproducir las características de un evento isquémico cardiaco agudo mediante el método de corazón aislado de Langendorff, la cual se podría traducir en una herramienta que funcione para búsqueda de nueva terapias para el tratamiento de esta enfermedad. Este estudio concluye que, bajo las condiciones de nuestro laboratorio, cuando se perfunde a una velocidad de $10,5 \mathrm{~mL} / \mathrm{min}$ la viabilidad del corazón se mantiene durante el tiempo del experimento. Ademas, la privacion del oxigeno al tejido cardiaco logra inducir una isquemia significativa que se refleja en un $44 \%$ de area de infarto obtenida con este protocolo. La puesta a punto de esta técnica nos ha permitido establecer una nueva linea de investigacion de Ciencias Biomédicas con enfasis en la Farmacología cardiovascular, que buscará aportar información que se extrapole al manejo y terapéutica de las cardiopatías isquémicas en nuestro país.

\section{Bibliografía}

[1] O. Langendorff, "Untersuchungen am überlebenden Säugethierherzen," Pflüger, Arch. Für Die Gesammte Physiol. Des Menschen Und Der Thiere, vol. 61, no. 6, pp. 291-232, 1895, doi: doi:10.1007/bf01812150.

[2] H. G. Zimmer, "Modifications of the isolated frog heart preparation in Carl Ludwig's Leipzig Physiological Institute: Relevance for cardiovascular research," Can. J. Cardiol., vol. 16, no. 1, 2000.

[3] M. Skrzypiec-Spring, B. Grotthus, A. Szelag, and R. Schulz, "Isolated heart perfusion according to Langendorff-Still viable in the new millennium," J. Pharmacol. Toxicol. Methods, vol. 55, no. 2, pp. 113-126, 2007, doi: 10.1016/j. vascn.2006.05.006.

[4] H. J. Döring, "The isolated perfused heart according to Langendorff technique--function--application," Physiol. Bohemoslov., vol. 39, no. 6, p. 481-504, 1990, [Online]. Available: http://europepmc.org/abstract/MED/2103635.

[5] R. M. Bell, M. M. Mocanu, and D. M. Yellon, "Retrograde heart perfusion: The Langendorff technique of isolated heart perfusion,” J. Mol. Cell. Cardiol., vol. 50, no. 6, pp. 940-950, 2011, doi: 10.1016/j.yjmcc.2011.02.018.

[6] D. J. Herr, E. S. Aune, and D. R. Menick, "Induction and assessment of ischemia-reperfusion injury in langendorffperfused rat hearts," J. Vis. Exp., vol. 2015, no. 101, pp. 1-7, 2015, doi: 10.3791/52908.

[7] W. D. Ito et al., "Infarct size measurement by triphenyltetrazolium chloride staining versus in vivo injection of propidium lodide," J. Mol. Cell. Cardiol., vol. 29, no. 8, pp. 2169-2175, 1997, doi: 10.1006/jmcc.1997.0456.

[8] Q. Liu et al., "Hydromorphine postconditioning protects isolated rat heart against ischemia-reperfusion injury via activating P13K/Akt/eNOS signaling," Cardiovasc. Ther., vol. 36, no. 6, pp. 1-9, 2018, doi: 10.1111/17555922.12481.

[9] Z. Tang et al., "Taxifolin protects rat against myocardial ischemia/reperfusion injury by modulating the mitochondrial apoptosis pathway," PeerJ, vol. 2019, no. 1, pp. 1-17, 2019, doi: 10.7717/peerj.6383.

[10] J. Xiao, Z. P. Ke, Y. Shi, Q. Zeng, and Z. Cao, "The cardioprotective effect of thymoquinone on ischemia-reperfusion injury in isolated rat heart via regulation of apoptosis and autophagy," J. Cell. Biochem., vol. 119, no. 9, pp. 72127217, 2018, doi: 10.1002/jcb.26878.

[11] A. M. Petkovic et al., "The Effects of Potassium Cyanide on the Functional Recovery of Isolated Rat Hearts after Ischemia and Reperfusion: The Role of Oxidative Stress," Oxid. Med. Cell. Longev., vol. 2018, 2018, doi: $10.1155 / 2018 / 5979721$. 


\section{Agradecimientos}

Al Sistema Nacional de Investigación (Investigador Nacional/ Estela Guerrero), al financiamiento recibido por la Vicerrectoría de la Universidad de Panamá (VIP-01-07-072021-03) y la Secretaría Nacional de Ciencia, Tecnología e Innovación (PAAC-NI-2020-II-29).

\section{Autorización y Licencia CC}

Los autores autorizan a APANAC XVIII a publicar el artículo en las actas de la conferencia en Acceso Abierto (Open Access) en diversos formatos digitales (PDF, HTML, EPUB) e integrarlos en diversas plataformas online como repositorios y bases de datos bajo la licencia CC:

Attribution-NonCommercial-ShareAlike 4.0 International (CC BY-NC-SA 4.0) https://creativecommons. org/licenses/by-nc-sa/4.0/.

Ni APANAC XVIII ni los editores son responsables ni del contenido ni de las implicaciones de lo expresado en el artículo. 